\title{
ii- Open Set in Bitopological Spaces
}

\section{Beyda S. Abdullah}

baedaa419@gmail.com
Amir A. Mohammed

amirabdulillah64@gmail.com

Department of Mathematics,

College of Education for Pure Sciences,

University of Mosul, Mosul, Iraq.

Received on: 21/01/2020

Accepted on: 07/05/2020

\section{ABSTRACT}

In this paper, we define $i i$-open set in bitopological space as follows: Let $\left(X, \tau_{1}, \tau_{2}\right)$ be a bitopological space, a subset $A$ of $X$ is said to be $\left(\tau_{1} \tau_{2}-i i\right.$ - open set $)$ if there exist $U, V \neq \varnothing, X$ and $U, V \in \tau_{1} \cup \tau_{2}$ such that:

1. $A=\operatorname{int}^{1}(U) \quad$ or $A=\operatorname{int}^{2}(V)$

2. $A \subseteq C L^{1}(A \cap U) \quad$ or $A \subseteq C L^{2}(A \cap V)$

We study some characterizations and properties of this class. Also, we explain the relation between $i i$ - open sets and open sets, $i$-open sets and $\alpha$-open sets in bitopological space. Furthermore, we define $i i_{\text {- }}$ continuous mapping on bitopological spaces with some properties.

Keywords: $\alpha$ - open set, $i$ - open set, $i i$ - open set, bitopological space.

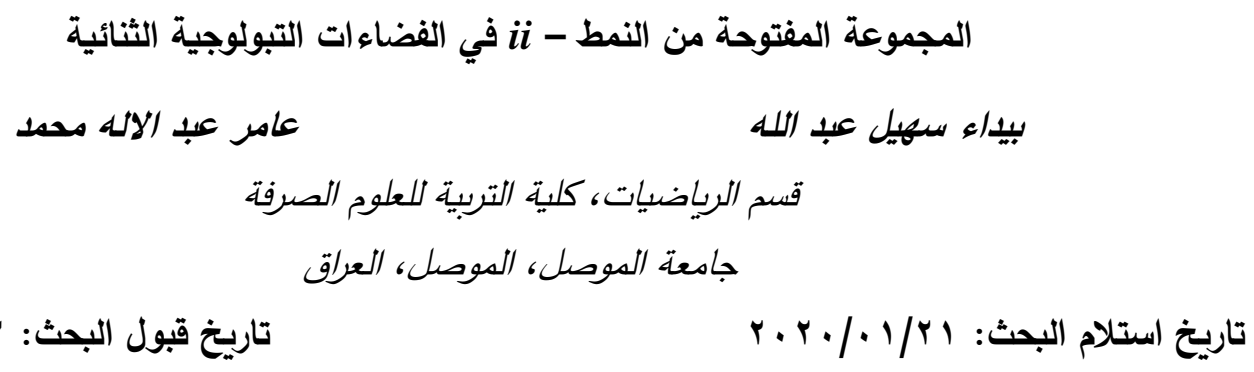

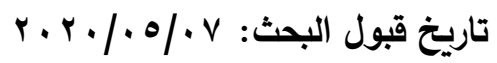

| (الملخص

في هذا البحث، عرفنا المجموعة المفتوحة من النمط - ii في الفضاء التبولوجي الثنائي على النحو التالي:

ليكن النمط-

1. $A=\operatorname{int}^{1}(U)$

or $\quad A=$ int $^{2}(V)$

2. $A \subseteq C L^{1}(A \cap U)$

or $\quad A \subseteq C L^{2}(A \cap V)$

درسنا بعض الصفات والخواص لهذا الصنف. ايضا وضحنا العلاقة بين المجاميع المفتوحة من النمط -

والمجاميع المفتوحة، المجاميع المفتوحة من النمط - i والمجاميع المفتوحة من النمط-م في الفضاء التبولوجي الثنائي. اضافة الى ذلك، عرفنا التطبيق المستمر من النمط - ii على الفضاءات التبولوجية الثنائية مع بعض ون فئه

الكلمات المفتاحية: مجموعة مفتوحة من النمط -ه ، مجموعة مفتوحة من النمط -i ، مجموعة مفتوحة من النمط

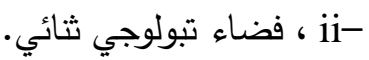




\section{Introduction}

The concept of bitopological space was introduced by Kelly [5] in 1963. A set equipped with two topologies is called a bitopological space and is denoted by $\left(X, \tau_{1}\right.$, $\left.\tau_{2}\right)$, where $\left(X, \tau_{1}\right),\left(X, \tau_{2}\right)$ are two topologicals spaces. Since then many authors have contributed to the development of various bitopological properties. Mohammed and Abdullah in 2019 [1], introduced the concept of $i i$-open sets as follows: A subset $A$ of a topological space $(X, \tau)$ is said to be $i i$-open set if there exists an open set $G$ in the topology of $X$, such that: $G \neq \emptyset, X, \quad A \subseteq C L(A \cap G)$ and $\operatorname{int}(A)=G$. later, the same authors in [2] studied topological properties of $i i$-derived, $i i$-border, $i i$-frontier, $i i$ exterior of a set by using the concept of $i i$-open sets. It is shown in [1] that each of $\tau \subset \tau^{i i}$ (the family of all ii-open sets) and $\tau^{i i}$ is a topology on $X$. Following Mahdi in [4] and Askandar and Mohammed in [3], we introduce the concept of ii-open set in bitopological space as follows: Let $\left(X, \tau_{1}, \tau_{2}\right)$ be a bitopological space, a subset $A$ of $X$ is said to be $\left(\tau_{1} \tau_{2}-i i\right.$ - open set $)$ if there exist $U, V \neq \varnothing, X$ and $U, V \in \tau_{1} \cup \tau_{2}$ such that:

$$
\begin{aligned}
& \text { 1. } A=\operatorname{int}^{1}(U) \\
& \text { 2. } A \subseteq C L^{1}(A \cap U) \\
& \text { or } \quad A=i n t^{2}(V) \\
& \text { or } A \subseteq C L^{2}(A \cap V)
\end{aligned}
$$

The aim of this paper is to study $\tau_{1} \tau_{2}-i i$ - open set with their properties and characterizations in bitopological space $\left(X, \tau_{1}, \tau_{2}\right)$. Further, we study the comparison of this class with other classes namely: $\tau_{1} \tau_{2}-$ open, $\tau_{1} \tau_{2}-i$ - open and $\tau_{1} \tau_{2}-\alpha$ open sets. This work consists of three sections. In the first one, we define ii-open sets in bitopological spaces and we give many related examples. In the second section, we discuss the relationship between $i i$-open sets with open, $i$-open and $\alpha$-open sets in bitopological spaces. In the third section, we define ii-continuous mapping on bitopological spaces and we study the relation between this type of mapping with continuous, $\alpha$-continuous [7] and $i$-continuous mapping [3] .

\section{1. ii-Open Sets in Bitopological Spaces}

In this section, we recall the following definitions, which are useful in the sequel.

Definition 1.1 [3,6,7]: Let $\left(X, \tau_{1}, \tau_{2}\right)$ be a bitopological space, a subset $A$ of $X$ is said to be

1. ( $\tau_{1} \tau_{2}$-open set ) [6] if $A \in \tau_{1} \cup \tau_{2}$

2. $\left(\tau_{1} \tau_{2}-\alpha\right.$ - open set $)$ [7] if $\quad A \subseteq \tau_{1}-\operatorname{int}\left(\tau_{2}-\operatorname{cl}\left(\tau_{1}-\operatorname{int}(A)\right)\right.$

3. $\left(\tau_{1} \tau_{2}-i\right.$ - open set ) [3] if there exists $\tau_{1}$ - open set $(G \neq \emptyset, X)$ such that $A \subseteq \tau_{2}-C L(A \cap G)$. The complement of $\left(\tau_{1} \tau_{2}-i\right.$ - open set $)$ is called $\left(\tau_{1} \tau_{2}-i\right.$ closed set ).

Definition 1.2: Let $\left(X, \tau_{1}, \tau_{2}\right)$ be a bitopological space, a subset $A$ of $X$ is said to be $\tau_{1} \tau_{2}-i i$ - open set if there exists $U, V \neq \varnothing, X$ and $U, V \in \tau_{1} \cup \tau_{2}$ such that:
1. $A=\operatorname{int}^{1}(U)$
or $A=\operatorname{int}^{2}(V)$
2. $A \subseteq C L^{1}(A \cap U)$
or $A \subseteq C L^{2}(A \cap V)$

The complement of $\tau_{1} \tau_{2}-i i$ - open set is called $\tau_{1} \tau_{2}-i i$ - closed set .

Example 1.1: Let $X=\{a, b, c\}, \tau_{1}=\{\varnothing, X,\{a\},\{b\},\{a, b\}\}, \tau_{2}=\{\varnothing, X,\{a\},\{c\},\{a, c\}\}$

$$
\begin{gathered}
\tau_{1} \cup \tau_{2}=\{\varnothing, X,\{a\},\{b\},\{c\},\{a, b\},\{a, c\}\} \\
\tau_{1}-\text { closed sets are: }\{\varnothing,\{b, c\},\{a, c\},\{c\}, X\} \\
\tau_{2}-\text { closed sets are: }\{\varnothing,\{b, c\},\{a, b\},\{b\}, X\}
\end{gathered}
$$




$$
\begin{aligned}
& \text { 1. } A=\{a\} \quad U=\{a\} \quad V=\{a, b\} \\
& \{a\}=\operatorname{int}^{1}\{a\}=\{a\} \quad\{a\} \subseteq C L^{1}(\{a\} \cap\{a\})=\{a, c\} \\
& \text { 2. } A=\{b\} \quad U=\{b\} \quad V=\{a, b\} \\
& \{b\}=\operatorname{int}^{1}\{b\}=\{b\} \quad\{b\} \subseteq C L^{1}(\{b\} \cap\{b\})=\{b, c\} \\
& \text { 3. } A=\{c\} \quad U=\{c\} \quad V=\{a, c\} \\
& \{c\}=\operatorname{int}^{1}\{c\}=\varnothing \quad\{c\}=\operatorname{int}^{2}\{c\}=\{c\} \\
& \{c\} \subseteq C L^{1}(\{c\} \cap\{c\})=\{c\} \\
& \text { 4. } A=\{a, b\} \quad U=\{a, b\} \quad V=\{b\} \\
& \{a, b\}=\operatorname{int}^{1}\{a, b\}=\{a, b\} \quad\{a, b\} \subseteq C L^{1}(\{a, b\} \cap\{a, b\})=X \\
& \text { 5. } A=\{a, c\} \quad U=\{a, c\} \quad V=\{c\} \\
& \{a, c\}=\operatorname{int}^{1}\{a, c\} \neq\{a\} \quad\{a, c\}=\operatorname{int}^{2}\{a, c\}=\{a, c\} \\
& \{a, c\} \subseteq C L^{1}(\{a, c\} \cap\{a, c\})=\{a, c\} \\
& \text { 6. } A=\{b, c\} \quad U=\{c\} \quad V=\{b\} \\
& \{b, c\}=\operatorname{int}^{1}\{b, c\} \neq\{b\} \quad\{b, c\} \subseteq C L^{1}(\{b, c\} \cap\{b\})=\{b, c\} \\
& \{b, c\}=\operatorname{int}^{2}\{b, c\} \neq\{c\} \\
& \tau_{1} \tau_{2}-i i-\text { open sets are: }\{\varnothing, X,\{a\},\{b\},\{c\},\{a, b\},\{a, c\}\} \\
& \tau_{1} \tau_{2}-i i-\text { closed sets are: }\{\varnothing, X,\{b, c\},\{a, c\},\{a, b\},\{c\},\{b\}\}
\end{aligned}
$$

Example 1.2: Let $X=\{a, b, c, d\}$,

$$
\begin{aligned}
& \tau_{1}=\left\{\varnothing, X,\{a\},\{b, c, d\}, \tau_{2}=\{\varnothing, X,\{a\},\{c\},\{a, c\}\}\right. \\
& \tau_{1} \cup \tau_{2}=\{\varnothing, X,\{a\},\{c\},\{a, c\},\{b, c, d\}\}
\end{aligned}
$$

$\tau_{1}-$ closed sets are: $\{\varnothing, X,\{b, c, d\},\{a\}\}$

$\tau_{2}-$ closed sets are: $\{\varnothing, X,\{b, c, d\},\{a, b, d\},\{b, d\}\}$. By using the definition (1.2) we get the $\tau_{1} \tau_{2}-i i$-open sets are: $\{\varnothing, X,\{a\},\{c\},\{a, c\},\{b, c, d\}\}$

\section{Some Properties of ii-Open Sets in Bitopological Spaces.}

Definition 2.1: Let $\left(X, \tau_{1}, \tau_{2}\right)$ be a bitopological space and let $A$ be a subset of $X$. Recall that:

1. The intersection of all $i i$-closed sets containing $A$ is called $i i$-closure of $A$, denoted by $\tau_{1} \tau_{2}-\operatorname{clii}(A)$.

2. The union of all $i i$-open sets contained in $A$ is called $i i$-interior of $A$, denoted by

$$
\tau_{1} \tau_{2}-\operatorname{intii}(A) \text {. }
$$

Theorem 2.2: Let $\left(X, \tau_{1}, \tau_{2}\right)$ be a bitopological space and $A \subseteq X$ then, the following are true:

1. $\tau_{1} \tau_{2}-\operatorname{intii}(A)$ is $\tau_{1} \tau_{2}-i i-$ open set.

2. $\tau_{1} \tau_{2}-\operatorname{clii}(A)$ is $\tau_{1} \tau_{2}-i i-$ closed set.

3. If $A$ is $\tau_{1} \tau_{2}-i i-$ closed then, $A=\tau_{1} \tau_{2}-\operatorname{clii}(A)$.

4. If $A$ is $\tau_{1} \tau_{2}-i i-$ open then, $A=\tau_{1} \tau_{2}-\operatorname{intii}(A)$.

5. If $A \subset B$ then, $\tau_{1} \tau_{2}-\operatorname{clii}(A) \subset \tau_{1} \tau_{2}-\operatorname{clii}(B)$.

6. $X-\tau_{1} \tau_{2}-\operatorname{clii}(A)=\tau_{1} \tau_{2}-\operatorname{intii}(X-A)$.

7. $X-\tau_{1} \tau_{2}-\operatorname{intii}(A)=\tau_{1} \tau_{2}-\operatorname{clii}(X-A)$.

8. $x \in \tau_{1} \tau_{2}-\operatorname{clii}(A)$ iff for all $i i$-open set $G$ containing $A, A \cap G \neq \emptyset$.

Proof: (1), (2), (3), (4) it follows from the definition (1.2). 
5. Let $A \subset B$. Since $\tau_{1} \tau_{2}-\operatorname{clii}(A)$ is the smallest $i i$-closed set containing $A$, then $A \subset$ $\tau_{1} \tau_{2}-\operatorname{clii}(A)$, So $B \subset \tau_{1} \tau_{2}-\operatorname{clii}(B)$. We get $\tau_{1} \tau_{2}-\operatorname{clii}(A) \subset \tau_{1} \tau_{2}-$ $\operatorname{clii}(B)$.

6. $X \in X-\tau_{1} \tau_{2}-\operatorname{clii}(A)$ if $x \notin X-\tau_{1} \tau_{2}-\operatorname{clii}(A)$, then for all $G \in \tau_{1} \tau_{2}-\operatorname{iio}(X)$ containing $x$, then $A \cap G \neq \emptyset$ if and only if $x \in G \subset X-A$ if and only if $x \in \tau_{1} \tau_{2}-$ intii $(X-A)$.

7. In a similar way to proof (6).

8. Let $x \in \quad \tau_{1} \tau_{2}-\operatorname{clii}(A)$ we shall prove that $A \cap G \neq \emptyset$ for all $i i$-open set $\mathrm{G}$ containing $x$. Let there exists set $G$ is $\tau_{1} \tau_{2}-i i$-open containing $x$ such that $A \cap G=\emptyset$, so $A$ $\subseteq G^{c}$ and $G^{c}$ is $\tau_{1} \tau_{2}-i i-$ closed set, therefore $\tau_{1} \tau_{2}-\operatorname{clii}(A) \subset \tau_{1} \tau_{2}-\operatorname{clii}\left(G^{c}\right)$. Since $x \in \tau_{1} \tau_{2}-\operatorname{clii}(A)$ this implies that $x \in \tau_{1} \tau_{2}-\operatorname{clii}\left(G^{c}\right)$. Since $G^{c}$ is $\tau_{1} \tau_{2}-$ $i i$ - closed set by theorem(2.2)(3) $x \in G^{c}$ means that $x \notin G$. Therefore $A \cap G \neq \emptyset$ for all $\tau_{1} \tau_{2}$-open sets $\mathrm{G}$ containing $x$.

Converse: Let $A \cap G \neq \emptyset$ for all $G$ is $\tau_{1} \tau_{2}-i i$ - open set containing $x$, we shall prove that $x \in \tau_{1} \tau_{2}-\operatorname{clii}(A)$. Let $x \notin \tau_{1} \tau_{2}-\operatorname{clii}(A)$ by definition (2.1), there exists $\tau_{1} \tau_{2}-i i-$ closed set $\mathrm{F}$ containing $A$ such that $x \notin F$, so $x \in F^{c}$ and $F^{c}$ is $\tau_{1} \tau_{2}-i i$-open set. Therefore $A \cap F^{c}=\emptyset$. This implies a contradiction. Therefore $x \in \tau_{1} \tau_{2}-\operatorname{clii}(A)$.

Theorem 2.3: Every open set in bitopological space $\left(X, \tau_{1}, \tau_{2}\right)$ is $\tau_{1} \tau_{2}-i i$-open

Proof: Let $G$ be open set in bitopological space $\left(X, \tau_{1}, \tau_{2}\right)$ :

1. If $G \in \tau_{1}$ we shall prove that $G$ is $\tau_{1} \tau_{2}-i i-$ open. Put $U=G$, we get

i. $G \subseteq C L^{1}(G \cap G) \subseteq C L^{1}(G)$

ii. $G=$ int $^{l}(G) \quad$ because $G \in \tau_{1}$

2. If $G \in \tau_{2}$, put $V=G$, we get

iii. $G \subseteq C L^{2}(G \cap G) \subseteq C L^{2}(G)$

iv. $G=$ int $^{2}(G) \quad$ because $G \in \tau_{2}$

By using the definition (1.2) we get in both cases $G$ is $\tau_{1} \tau_{2}-i i-$ open set. The converse of the above theorem is not true in general as shown in the following example:

Example 2.4: Let $X=\{a, b, c\}, \tau_{1}=\left\{\varnothing, X,\{b\},\{a, c\}, \tau_{2}=\{\varnothing, X,\{c\},\{a, b\}\}\right.$

$\tau_{1} \cup \tau_{2}=\{\varnothing, X,\{b\},\{c\},\{a, c\},\{a, b\}\}$

$\tau_{1}-$ closed sets are: $\{\varnothing, X,\{a, c\},\{b\}\}$

$\tau_{2}-$ closed sets are: $\{\varnothing, X,\{c\},\{a, b\}\}$. By using the definition (1.2) we get the $\tau_{1} \tau_{2}-$ $i i-$ open sets are: $\{\varnothing, X,\{a\},\{b\},\{c\},\{a, c\},\{a, b\}\}$.

We see that $A=\{a\}$ is $\tau_{1} \tau_{2}-i i-$ open set but $A$ is not $\tau_{1} \tau_{2}$-open set.

Remark 2.5: From the definition (1.2) we get every $\tau_{1} \tau_{2}-i i$-open set in bitopological space is $\tau_{1} \tau_{2}-i$-open set. The converse of the above remark is not true in general as shown in the following example:

Example 2.6: Let $X=\{a, b, c\}, \tau_{1}=\left\{\varnothing, X,\{a\}, \tau_{2}=\{\varnothing, X,\{a\},\{a, b\}\}\right.$

$\tau_{1} \cup \quad \tau_{2}=\{\varnothing, X,\{a\},\{a, b\}\}$

$\tau_{1}-$ closed sets are: $\{\varnothing, X,\{b, c\}\}$

$\tau_{2}-$ closed sets are: $\{\varnothing, X,\{b, c\},\{c\}\}$

$\tau_{1} \tau_{2}-i$-open sets are: $\{\varnothing, X,\{a\},\{a, b\},\{a, c\}\}$

$\tau_{1} \tau_{2}-i i-$ open sets are: $\{\varnothing, X,\{a\},\{a, b\}\}$

$A=\{a, c\}$ is $\tau_{1} \tau_{2}-i$-open set but $A$ is not $\tau_{1} \tau_{2}-i i-$ open set. 
Remark 2.7: We see that every $\tau_{1} \tau_{2}-\alpha$-open set in bitopological space is not necessary $\tau_{1} \tau_{2}-i i-$ open set, but this relation is true in topological spaces, that is, every $\alpha$-open set in topological space is $i i-$ open set Theorem(2.12)[1]. Also, we see that every

$\tau_{1} \tau_{2}-i i-$ open set in bitopological space is not necessary $\tau_{1} \tau_{2}-\alpha$-open set, it means that:

$\tau_{1} \tau_{2}-\alpha$-open set $\longleftrightarrow \tau_{1} \tau_{2}-i i-$ open set

As in the following examples:

\section{Example 2.8:}

Let $X=\{a, b, c, d\}, \tau_{1}=\left\{\varnothing, X,\{a\},\{b, c, d\}, \tau_{2}=\{\varnothing, X,\{a\},\{c\},\{a, c\}\}\right.$

$\tau_{1} \cup \tau_{2}=\{\varnothing, X,\{a\},\{c\},\{a, c\},\{b, c, d\}\}$

$\tau_{1}-$ closed sets are: $\{\varnothing, X,\{b, c, d\},\{a\}\}$

$\tau_{2}-$ closed sets are: $\{\varnothing, X,\{b, c, d\},\{a, b, d\},\{b, d\}\}$

$\tau_{1} \tau_{2}-i i-$ open sets are: $\{\varnothing, X,\{a\},\{c\},\{a, c\},\{b, c, d\}\}$

$\tau_{1} \tau_{2}-\alpha$-open sets are: $\{\varnothing, X,\{a\},\{b, c, d\}\}$

$A=\{c\}$ is $\tau_{1} \tau_{2}-i i-$ open set but $A$ is not $\tau_{1} \tau_{2}-\alpha$-open set

Example 2.9: Let $X=\{a, b, c\}, \tau_{1}=\{\varnothing, X,\{a\}\}, \quad \tau_{2}=\{\varnothing, X,\{a\},\{a, b\}\}$

$\tau_{1} \cup \tau_{2}=\{\varnothing, X,\{a\},\{a, b\}\}$

$\tau_{1}-$ closed sets are: $\{\varnothing, X,\{b, c\}\}$

$\tau_{2}-$ closed sets are: $\{\varnothing, X,\{b, c\},\{c\}\}$

$\tau_{1} \tau_{2}-i i-$ open sets are: $\{\varnothing, X,\{a\},\{a, b\}\}$

$\tau_{1} \tau_{2}-\alpha$-open sets are: $\{\varnothing, X,\{a\},\{a, b\},\{a, c\}\}$

$A=\{a, c\}$ is $\tau_{1} \tau_{2}-\alpha$-open set but $A$ is not $\tau_{1} \tau_{2}-i i$-open set.

Remark 2.10: From the above results we get the following diagram.

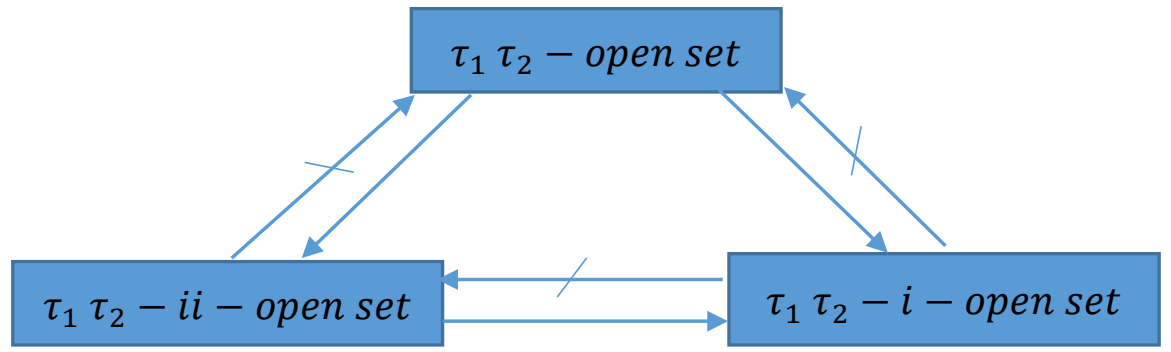

\section{3. ii- Continuity on Bitopological Spaces.}

In this section, we define $i i$-continuous mapping from $\left(X, \tau_{1}, \tau_{2}\right)$ in $\left(Y, \delta_{1}, \delta_{2}\right)$ by using the definition of $i i$-open sets in topological space $(X, \tau)$ see[1],[2] . Also, we study the relation between $i i$-continuous mapping on bitopological spaces with continuous and $i$-continuous mapping [3].

Definition 3.1: A mapping $f:\left(X, \tau_{1}, \tau_{2}\right) \rightarrow\left(Y, \delta_{1}, \delta_{2}\right)$ is called

1. Continuous if $f^{-1}(G)$ is $\tau_{1} \tau_{2}$-open set in $\left(X, \tau_{1}, \tau_{2}\right)$ for any open set $G$ in $\left(Y, \delta_{1}, \delta_{2}\right)$.

2. i-Continuous if $f^{-1}(\mathrm{G})$ is $\tau_{1} \tau_{2}-i$ - open set in $\left(X, \tau_{1}, \tau_{2}\right)$ for any open set $G$ in $\left(Y, \delta_{1}, \delta_{2}\right)$. 
Definition3.2: A mapping $f:\left(X, \tau_{1}, \tau_{2}\right) \rightarrow\left(Y, \delta_{1}, \delta_{2}\right)$ is called $i i$-continuous if $f^{-1}(\mathrm{G})$ is $\tau_{1} \tau_{2}-i i-$ open set in $\left(X, \tau_{1}, \tau_{2}\right)$ for any open set $G$ in $\left(Y, \delta_{1}, \delta_{2}\right)$.

Theorem 3.3: Let $f:\left(X, \tau_{1}, \tau_{2}\right) \rightarrow\left(Y, \delta_{1}, \delta_{2}\right)$ be mapping then:

1. Every continuous mapping is an $i i$-continuous,

2. Every $i i$-continuous mapping is an $i$-continuous.

Proof: (1) Let $G$ be an open set in $\left(Y, \delta_{1}, \delta_{2}\right)$. Since $f$ is continuous, it follows that $f^{-1}(\mathrm{G})$ is $\tau_{1} \tau_{2}$-open set in $\left(X, \tau_{1}, \tau_{2}\right)$. By Theorem (2.3). Hence $f^{-1}(\mathrm{G})$ is $\tau_{1} \tau_{2}-i i$-open set in $\left(X, \tau_{1}, \tau_{2}\right)$. Thus $f$ is ii-continuous.

(2) Let $G$ be open set in $\left(Y, \delta_{1}, \delta_{2}\right)$. Since $f$ is ii-continuous, it follows that $f^{-1}(G)$ is $\tau_{1} \tau_{2}-i i$-open set in $\left(X, \tau_{1}, \tau_{2}\right)$. By remark (2.5). Hence $f^{-1}(\mathrm{G})$ is $\tau_{1} \tau_{2}-i$-open set in $\left(X, \tau_{1}, \tau_{2}\right)$. Thus $f$ is $i$-continuous.

The converse of the above theorem is not true in general as shown in the following example:

Example 3.4: Let $X=Y=\{a, b, c\}, \tau_{1}=\left\{\varnothing, X,\{a\}, \tau_{2}=\{\varnothing, X,\{a\},\{a, b\}\}\right.$

$\delta_{1}=\{\varnothing, Y,\{a\},\{a, c\}\}, \quad \delta_{2}=\{\varnothing, Y,\{a, c\}\}$

$\tau_{1} \cup \tau_{2}=\{\varnothing, X,\{a\},\{a, b\}\}$

$\tau_{1}-$ closed sets are: $\{\varnothing, X,\{b, c\}\}$

$\tau_{2}-$ closed sets are: $\{\varnothing, X,\{b, c\},\{c\}\}$

$\tau_{1} \tau_{2}-i i-$ open sets are: $\{\varnothing, X,\{a\},\{a, b\}\}$

$\tau_{1} \tau_{2}-i$-open sets are: $\{\varnothing, X,\{a\},\{a, b\},\{a, c\}\}$

Let $f:\left(X, \tau_{1}, \tau_{2}\right) \rightarrow\left(Y, \delta_{1}, \delta_{2}\right)$ be the identity mapping then $f^{-1}(\{a\})=\{a\}, f^{-1}(\{b\})=\{b\}$, $f^{-1}(\{c\})=\{c\}$. Then $f$ is $i$-continuous on bitopological spaces but $f$ is not ii-continuous, Since $\{a, c\}$ is open set in $\left(Y, \delta_{1}, \delta_{2}\right) f^{-1}(\{a, c\})=\{a, c\}$ is not $\tau_{1} \tau_{2}-i i-$ open set in $\left(X, \tau_{1}, \tau_{2}\right)$.

\section{Conclusion 3.5:}

For future, also we can study the concept of $\tau_{1} \tau_{2}-i i-$ open mapping and $\tau_{1} \tau_{2}-i i$-homeomorphisem and other topological concepts between bitopological spaces $\left(X, \tau_{1}, \tau_{2}\right)$ and $\left(Y, \delta_{1}, \delta_{2}\right)$. 


\section{REFERENCES}

[1] Abdullah.B.S. and Mohammed A.A., (2019), ii- Open Sets in Topological Space, Internatinal Mathematical Forum, Vol.14, No.1, 41-48.

https:// doi.org/10.12988/imf.2019.913

[2] Abdullah.B.S. and Mohammed A.A., (2019), On Standard Concept Using ii-Open Sets, Open Access Journal, Vol 6, July 15. http:// dx.doi.org/10.4236/***2019***

[3] Askander.S.W. and Mohammed A.A., (2018), i-Open Sets in Topological Spaces, Al-Rafidain Journal of Computer Sciences and Math, Vol 12, No.1,13-23.

[4] Mahdi,Y.K.,(2007), Semi-Open and Semi-Closed Sets in Bitopological Spaces, Accepted in The First Science Conference of Education College, Babylon Unive,February 18-19.

[5] Kelly.j.c, (1963), Bitopological Spaces, London Math. Soc., Vol. 13, 71-89.

[6] Lellis Thivagav,M. and B.Meera.Devi, (2010), Bitopological B-Open Sets, International Journal of Algorithms, Computing and Math, Vol. 3,No. 3

[7] Lellis Thivagav,M., (1991), Generalization of Pairwise $\alpha$-Continuous Functions, Pure and Appl. Mathematica sci, Vol.28, 55-63. 